\title{
Required Time of Arrival as a Control Mechanism to Mitigate Uncertainty in Arrival Traffic Demand Management
}

\author{
Hyo-Sang Yoo, Christoph Mohlenbrink, Connie \\ Brasil, Nathan Buckley, Al Globus \\ San Jose State University / NASA Ames Research Center \\ Moffett Field, CA, U.S.A. \\ Hyo-Sang.Yoo@nasa.gov
}

\author{
Nancy M. Smith, Paul U. Lee \\ NASA Ames Research Center \\ Moffett Field, CA, U.S.A
}

\begin{abstract}
-the objective of this study is to explore the use of Required Time of Arrival (RTA) capability on the flight deck as a control mechanism on arrival traffic management to improve traffic delivery accuracy by mitigating the effect of traffic demand uncertainty. The uncertainties are caused by various factors, such as departure error due to the difference between scheduled departure and the actual take-off time. A simulation study was conducted using the Multi Aircraft Control System (MACS) software, a comprehensive research platform developed in the Airspace Operations Laboratory (AOL) at NASA Ames Research Center. The Crossing Time (CT) performance (i.e. the difference between target crossing time and actual crossing time) of the RTA for uncertainty mitigation during cruise phase was evaluated under the influence of varying two main factors: wind severity (heavy wind vs. mild wind), and wind error (1 hour, 2 hours, and 5 hours wind forecast errors). To examine the CT performance improvement made by the RTA, the comparison to the CT of the aircraft that were not assigned with RTA (NonRTA) under the influence of the selected factors was also made. The Newark Liberty International Airport (EWR) was chosen for this study. A total 66 inbound traffic to the EWR (34 of them were airborne when the simulation was initiated, 32 were predepartures at that time) was simulated, where the pre-scripted departure error was assigned to each pre-departure $(61 \%$ conform to their Expected Departure Clearance Time, which is $+/-300$ seconds of their scheduled departure time). The results of the study show that the delivery accuracy improvement can be achieved by assigning RTA, regardless of the influence of the selected two factors (the wind severity and the wind information inaccuracy). Across all wind variances, 66.9\% (265 out of 396) of the CT performance of the RTA assigned aircraft was within +/60 seconds (i.e. target tolerance range) and $88.9 \%$ (352 out of 396) aircraft met $+/-300$ seconds marginal tolerance range, while only $33.6 \%$ (133 out of 396) of the Non-RTA assigned aircraft's CT performance achieved the target tolerance range and $75.5 \%$ (299 out of 396) stayed within the marginal. Examination of the impact of different error sources - i.e. departure error, wind severity, and wind error - suggest that although large departure errors can significantly impact the $\mathrm{CT}$ performance, the impacts of wind severity and errors were modest relative the targeted +/60 second conformance range.
\end{abstract}

Keywords - Required Time of Arrival (RTA); Departure Error; Uncertainty Mitigation; Traffic Flow Management; TFMS; Integrated Demand Management (IDM)

\section{INTRODUCTION}

NASA has begun development of a near- to mid-term concept called "Integrated Demand Management" (IDM), which focuses on developing methods to effectively manage the arrival traffic demand to the available capacity. Under IDM, Traffic Flow Management System (TFMS) flowmanagement capabilities are used to strategically pre-condition demand into the more tactical Time-Based Flow Management (TBFM) system. One factor that can limit the effectiveness of this TFMS preconditioning, however, is its reliance on departure time management and pre-departure route assignment to control when the aircraft will enter the TBFM region [1].

In current operations, TFMS controls the departure times to manage aircraft entry times at a downstream location, but after the aircraft are airborne, the demand is managed using milesin-trail restrictions, which are decoupled from the TFMS schedule. To overcome this limitation and allow continued management of airborne aircraft to the TFMS schedule, this paper explores the use of RTA as a potential control mechanism to improve delivery accuracy of traffic demand to their TFMS scheduled entry time into TBFM.

\section{IDM OVERVIEW}

The overall goal of the IDM concept is to preempt the potential mismatch between traffic demand and resource constrained capacity in an airspace by strategically preconditioning the traffic demand using TFMS to feed a more manageable demand flow into TBFM which then uses a time based schedule to deliver traffic to the final destination. Potential benefits of better coordinated demand management between the two systems include minimizing unanticipated excessive TBFM-assigned ground delay by preventing overhead flow from being saturated [2] and preserving airborne delay absorption capabilities during congested flow [3].

Newark Liberty International Airport (EWR) was selected as a testing environment for exploring this concept. EWR represents one of the airports that experiences the highest arrival delay in the NAS [4,5]. In addition, aircraft trying to depart for EWR from close-in (200-300 miles) airports (e.g. 
Dulles or Boston) frequently incur excessive departure ground delay as there is very limited room in the overhead stream for them to fit [2]. EWR also had a nice mix of international vs. domestic traffic, as well as aircraft from various origination airports that were both near and far from EWR.

\section{A. TFMS and TBFM Integration in IDM}

TFMS enables the Air Traffic Control System Command Center, Air Route Traffic Control Centers, Terminal Radar Approach Control facilities, and airline operators to work collaboratively to achieve demand and capacity balancing for constrained airports and airspace within the National Airspace System.

The IDM concept utilizes a newly deployed NextGen capability within TFMS called the Collaborative Trajectory Options Program (CTOP) [6]. One of the key features of this tool is its capability to define multiple airspace boundaries, called Flow Constrained Areas (FCAs), and use them to redistribute traffic demand across different flows both temporally and geographically. In the initial IDM concept, an FCA ring (as shown in Fig. 1) was placed approximately 40 nautical miles around EWR airport, at or near the TBFM arrival meter fix points. In IDM, CTOP was used to predict initial arrival demand at the FCA and assigned capacity limits to the FCA ring that matched the expected rate that the TBFM system was set to deliver and what was expected to be Airport Arrival Rate (AAR) for EWR.

Setting the FCA ring capacity limits in CTOP generated initial departure times for all inbound EWR pre-departures (excluding those that planned to depart within 30 minutes) while at the same time giving preference to airborne flights in order to reserve their slots. This approach allowed only manageable pre-conditioned traffic to be delivered to EWR while reserving utilizable capacity in the overhead stream for departures within TBFM. The CTOP-generated departure times were issued to the aircraft as Estimated Departure Clearance Times (EDCTs).

\section{B. Using RTA for Accurate Delivery of Traffic into TBFM}

Within CTOP, there is currently no mechanism to continue managing FCA crossing times after the aircraft are airborne. In order to manage the airborne aircraft into TBFM environment, additional CTOP boundaries called Flow Evaluate Area (FEAs) were constructed. Figure 1 shows the locations of the FEAs that are approximately $400 \mathrm{~nm}$ away from EWR. All flights that crossed the FEAs had a target FEA Crossing Time (CT) anchored to the initial CTOP arrival schedule.

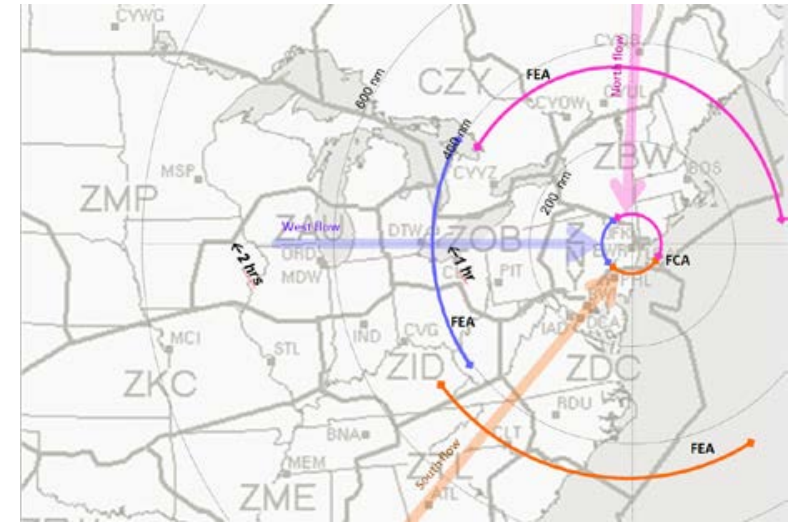

Fig. 1. FCA around EWR and FEAs used for the IDM concept.

Once the target CT was established for a given flight, the flight deck Flight Management System (FMS) Required Time of Arrival (RTA) feature could be used as an uncertainty mitigating control mechanism. After the aircraft reached cruise altitude (avoiding climb uncertainties) an RTA could be assigned in order to have the aircraft cross the FEA at its scheduled CT. The RTA capability, coupled to the CTOP FCA schedule, allowed the airborne aircraft to utilize the FMS to manage the conformance to their scheduled CTs.

\section{Uncertainties in Arrival Traffic}

The IDM concept assumes that if CTOP can generate an initial schedule to the FEA CTs and RTA could be utilized to deliver reasonable demand to TBFM, then the result would be better utilization of the available capacity that would reduce and distribute delays more effectively. However, one of challenges confronted by this concept is how to accurately deliver the traffic demand planned by TFMS into the TBFM environment. Various sources of uncertainties-e.g. aircraft performance modelling errors, airline/aircrew procedure modeling errors, wind severity and wind forecast error etc.can impede the accurate delivery of scheduled demand [7]. In addition, it is also unclear what the required delivery accuracy by CTOP needs to be in order to provide "reasonable" demand to TBFM.

One of the major sources of arrival demand uncertainty flowing into TBFM is manifested by departure error, which is the difference between scheduled and actual departure times. By looking at the actual departure errors of inbound aircraft to EWR from 10 sampled days in November, 2015 and December, 2015, it is identified that only about $69 \%$ of the departures from outside of the TBFM comply to their departure conformance time window (+/- 5 minutes of their EDCTscheduled departure times) and $85 \%$ of the departures within the TBFM region comply to their TBFM-departure conformance time window, which is 2 minute early and 1 minute late of the assigned take-off time.

Another source of uncertainty in accurate delivery is the errors associated with airborne aircraft. Any discrepancies between the estimated and the actual flight time to the CT once the aircraft is airborne would result in inaccurate delivery. Two potential sources of airborne errors were identified as wind strength and wind forecast error. The focus of this study, thus, 
lies on how much uncertainty mitigation of the aircraft can be achieved as they cross the FEAs by investigating how the CT performance improves with the use of RTA under varying wind conditions.

\section{METHOD}

\section{A. Simulation Environment}

The Multi-Aircraft Control System (MACS) software was used to explore the use of RTA. MACS provides a high fidelity air traffic control simulation environment, prototyping scheduling systems and simulating the air traffic [8].

In conjunction with MACS, a CTOP emulation, called nCTOP (NASA CTOP), was constructed to perform one of the key functions of the TFMS CTOP, which was to set capacity constraints to an FCA and automatically assigning delay to the pre-departures in order to balance the predicted arrival traffic demand at the FCA and its capacity limit [1]. The capacity at the FCA was set to be 44 aircraft per hour (11 aircraft per 15 min bin) in this study.

MACS FMS software emulates the general characteristics of a Honeywell "Black Label" FMS (B757/HW BL) with a user-adjustable RTA tolerance setting. This setting recomputes the flying speed so that aircraft crosses the target fix at the closest time to the CT, when the difference between the target CT and Estimated Time of Arrival (ETA) becomes greater than a certain parameter. The value of this parameter was set to be +/- 60 seconds as it was the desired target CT conformance tolerance for this study.

To simulate the effect of possible airline operators cost index preferences (i.e. the ratio of the time-related cost of an flight operation and the cost of fuel) on RTA acceptability, the speeds relevant to the cost index values used by several airlines were collected. Based on that information, general speed limitations were applied for aircraft weight classes to ensure that RTA-assigned aircraft's speeds would not exceed the operator-preferred flight speed windows (i.e. speed range that an airline would agree to fly). The resulting speed range for the "heavy" weight class aircraft was between Mach 0.76-0.85, and between Mach 0.74-0.84 for "large" weight class aircraft. The maximum speeds for each aircraft type were further constrained by their aircraft performance limits, thus the, which was below the upper limits of Mach 0.85 and Mach 0.84 for some aircraft types. The average initial cruise speed of the large aircraft when RTA was assigned was Mach 0.78. The minimum and maximum initial cruise speed before the RTA assignment was Mach 0.74 and Mach 0.82, respectively. For the heavy aircraft, the average initial speed was Mach 0.78. The minimum and maximum initial cruise speed was Mach 0.76 and Mach 0.83, respectively.

The traffic scenario was derived from actual recorded traffic from July 22, 2014. Several modifications were made based on the feedback from subject matter experts to have the most representative characteristics of the nominal operations into EWR during a clear weather day. The scenario lasted for 5 hours, and included a total of 66 aircraft which cross the FEAs and are eligible for the RTA assignment, 34 of which were airborne when the scenario was initiated. RTA-eligible aircraft had to be at cruise altitude and at least 200 miles from the FEAs. Traffic came from three basic arrival flows into EWR: North, South and West. North flow had a head wind generally and the South and West flows had tail winds (Figures 2 and 3).

\section{B. Experiment Design}

In this study, CT performance of RTA was evaluated under the influence of wind severity and wind error. A $2 \times 2 \times 3$ Factor experimental design consisted of three factors: the RTA conditions (RTA and Non-RTA), wind severity (mild and heavy wind), and wind error (1, 3, and 5 hours wind forecast errors).

\section{Wind Severity and Forecast Error}

For the wind data, National Oceanic and Atmospheric Administration's (NOAA's) 40 km resolution Rapid Refresh (RAP) file was used. A RAP wind file from January 11, 2014 12:00:00 Zulu was selected to represent the heavy wind condition, with the 1 hour forecast RAP wind file used as the "true wind" in the simulated environment. The 2 hours forecast wind was then used as the 1 hour forecast during the simulation, the 3 hours forecast wind was used as the 2 hours wind forecast, and the 6 hours forecast as the 5 hours forecast wind. The simulated environment and the ground scheduling system used the same "true wind" information, and only the wind information on the flight deck varied. Also, the wind files were kept static throughout the runs avoiding more variability to be induced.

The following Figure 2 shows the heavy wind condition at the $29951 \mathrm{ft}$. (isobaric pressure at $27500 \mathrm{pa}$ ).

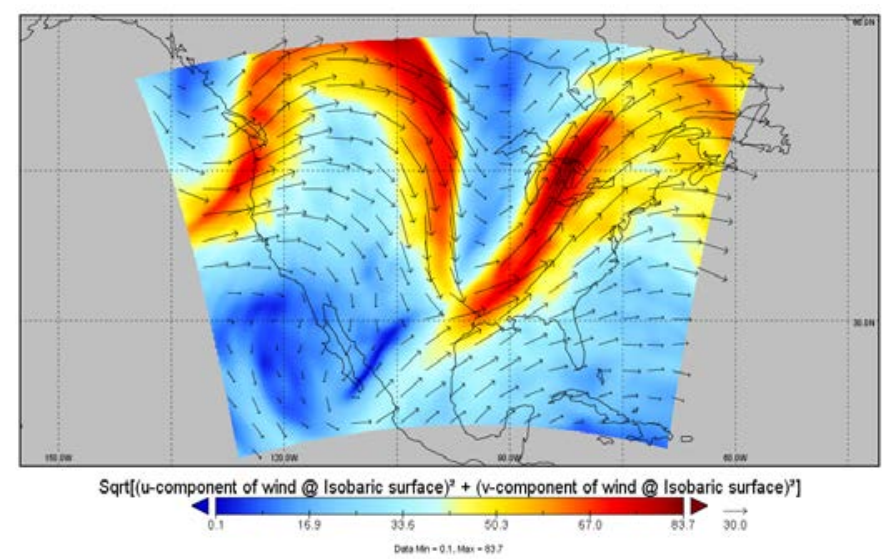

Fig. 2. Heavy wind condition $40 \mathrm{~km}$ resolution RAP winds $(\mathrm{m} / \mathrm{s})$ at 11 January-2014 12:00:00Z 27500 pa $(\approx 29951 \mathrm{ft})$

Wind severity can significantly affect the performance of RTA as it can change the ground speed of the aircraft. The RAP wind file (May 10, 2014) from a late spring season was chosen as the mild wind condition. The following Figure 3 represents the mild wind condition (May 10, 2014 11:00:00 Zulu) at $29951 \mathrm{ft}$. (isobaric pressure at $27500 \mathrm{pa}$ ). 


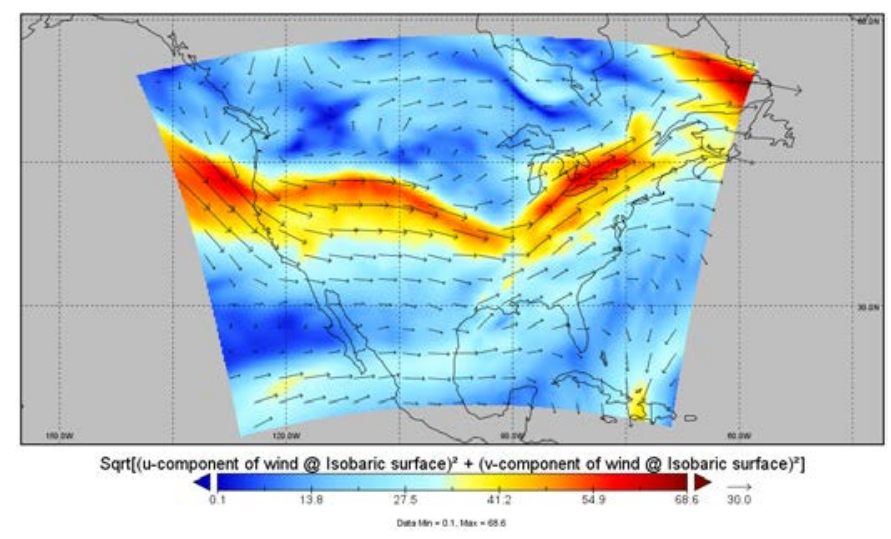

Fig. 3. Mild wind $40 \mathrm{~km}$ resolution RAP wind (m/s) at 10-May-2014 11:00:00Z 27500.0 pa $(\approx 29951.0 \mathrm{ft})$

The box-plots in Figure 4 show the mean wind speed across the whole nation at the different altitudes (10000, 20000, 30000 , and $40000 \mathrm{ft}$.). The wind speed is computed using the following equation (1):

$$
\text { Wind speed (knots) }=\sqrt{\frac{1}{N} \sum_{n=1}^{N}(u)^{2}+(v)^{2}}
$$

In the equation (1), $\mathrm{N}$ is the number of sampled observations, $u$ is the east-west component of the wind vector and $v$ is the north-south component.

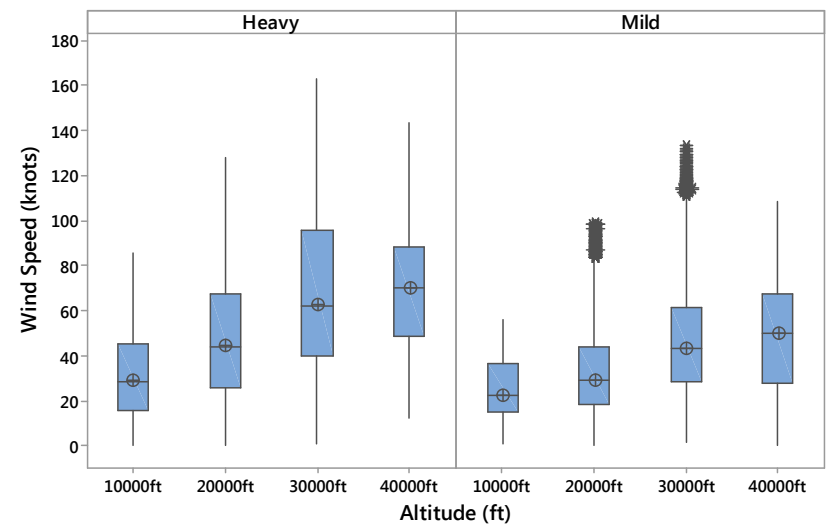

Fig. 4. Wind severity (mild vs. heavy) at the different altitudes

The impact of wind information accuracy relative to the true wind in the environment on the FMS and its time estimation has been identified in the past studies [9]. There are various types of wind error that could negatively affect the FMS operation, such as wind forecast resolution errors, wind blending errors in the process of merging forecast wind in FMS and actual wind being sensed outside of aircraft [10]. In this study, wind forecast errors for each wind severity condition were used as the major source of wind error that affected the wind information inaccuracy on FMS operations.

The wind forecast error of the selected heavy and mild wind condition were quantified using the following equation (2) [11]:
Wind forecast errors (RMSVE) $=\sqrt{\frac{1}{N} \sum_{n=1}^{N}\left(u_{f}-u_{0}\right)^{2}+\left(v_{f}-v_{0}\right)^{2}}$

In the equation (2), wind forecast errors are quantified as Root Mean Square Vector Error (RMSVE). $f$ and $o$ indicate forecast and observed (true wind in the simulated environment) wind respectively. Other notations are same as the equation (1).

Figures 5 \& 6 below show the wind forecast errors $(1,2$, and 5 hours forecast error) of the selected heavy and mild wind condition at different altitudes (10000, 20000, 30000, and $40000 \mathrm{ft}$.).

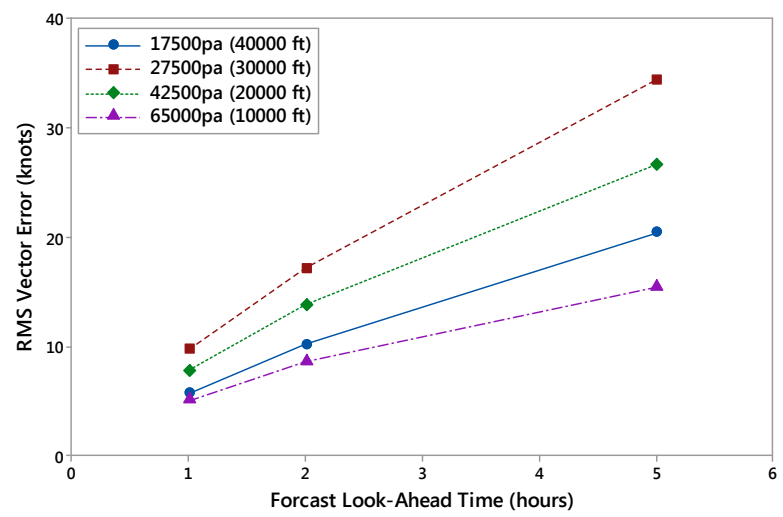

Fig. 5. Heavy wind: 1, 2, and 5 wind forecast errors.

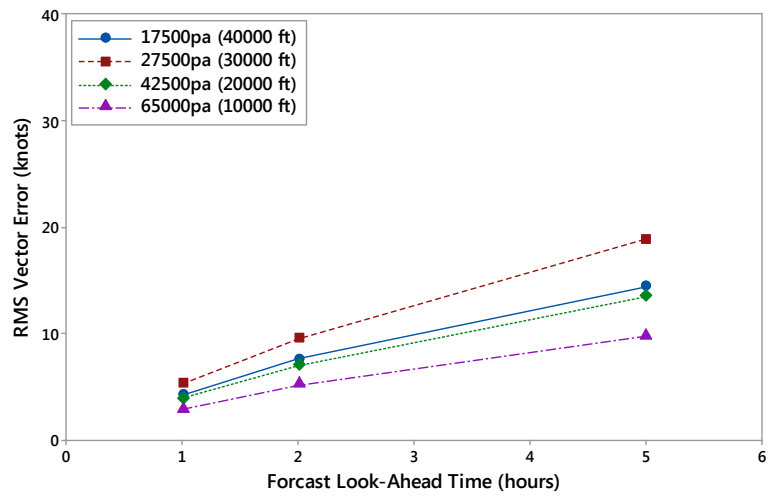

Fig. 6. Mild wind: 1, 2, and 5 wind forecast errors.

The range of the cruise altitude of the aircraft in the traffic scenario is between 33000 and $37000 \mathrm{ft}$. In Figures 5 and 6, it can be determined that wind forecast errors increase as the forecast look ahead time becomes greater for that cruise altitude ranges.

\section{Departure Error}

In this study, pre-scripted departure errors were generated by randomly drawing the departure errors from the distribution of errors in the real traffic data. The errors were kept static between all runs and conditions. Figure 7 shows the 
departure errors of the 32 pre-departures that crossed the FEAs and eligible for the RTA assignment. $61 \%$ of the predepartures conformed to their EDCT departure time (+/-300 seconds of its scheduled departure time). The average of the departure errors was 2.4 seconds and median was -75.0 seconds. The minimum and maximum were -906.0 seconds and 1129.0 seconds, respectively.

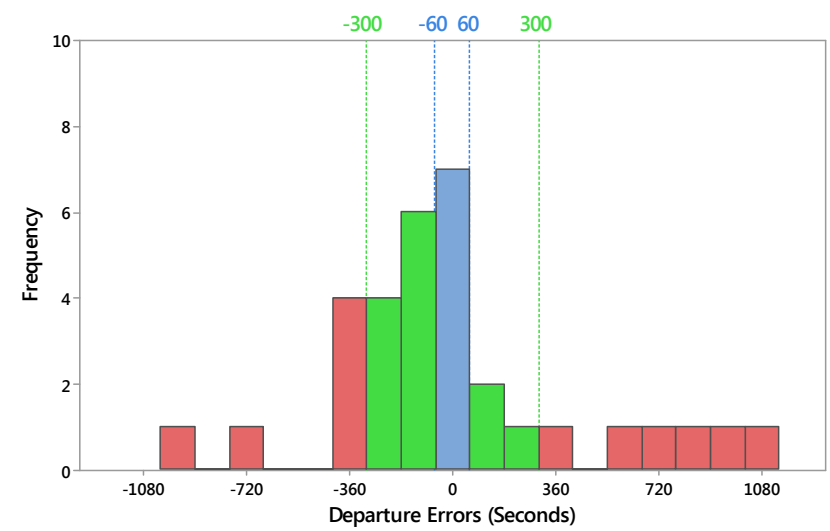

Fig. 7. Pre-scripted departure errors (seconds) of the pre-departures that crossed the FEAs.

\section{E. Dependent Variable}

The dependent variable of this study is the Crossing Time (CT) performance, which is the target CT at the FEA minus the actual CT. Hence, a negative error indicates that the flight was late to its target $\mathrm{CT}$, and a positive error indicates that it arrived early to its target CT. The CT performance was measured for all aircraft, whether or not they were assigned an RTA.

\section{HYPOHESES}

The following hypotheses were examined to explore the use of RTA to improve delivery accuracy at the FEAs to see how performance varies under the influence of identified factors.

A. Hypothesis A: Assigning RTA to aircraft will improve Crossing Time performance.

B. Hypothesis B: Strong winds may degrade Crossing Time performance.

C. Hypothesis C: Flight distance may affect Crossing Time performance.

D. Hypothesis D: Crossing Time performance of RTA assigned aircraft will decrease as wind forecast errors increase.

Hypothesis A compares the CT performance of RTA assigned aircraft to Non-RTA assigned aircraft. Hypothesis B examines how wind severity affects the CT performance of RTA and Non-RTA assigned aircraft. Hypothesis C examines the effect of flight distance (the distance from cruise phase until the aircraft crosses the FEA) on the CT performance of both RTA and Non-RTA aircraft. Hypothesis D provides insight on wind forecast accuracy requirements for achieving good CT performance by identifying the relationship between wind forecast errors and CT performance.

\section{RESUlts}

A. Results of Hypothesis A: Assigning RTA to aircraft improves Crossing Time performance.

A total of 396 aircraft (both pre-departures and airborne) received RTA across all conditions during the study. Of those, 265 (66.9\%) aircraft crossed the FEAs within +/- 60 seconds (i.e. the targeted tolerance range for this study) and 352 (88.9\%) aircraft crossed the FEAs within +/-300 seconds, which was identified as a marginal tolerance range. In contrast, only $33.6 \%$ (133 out of 396) of the Non-RTA assigned aircraft's CT performance met the targeted tolerance range and $75.5 \%$ (299 out of 396) crossed the FEAs within the marginal tolerance range. The marginal range of $+/-5$ minutes was established based on subject matter experts saying that 5minute conformance was "workable/marginal" for the TBFM system to manage the arrival flow into EWR. Figure 8 shows the histogram of the CT performance of RTA and Non-RTA assigned aircraft. The table I summarizes the CT performance.

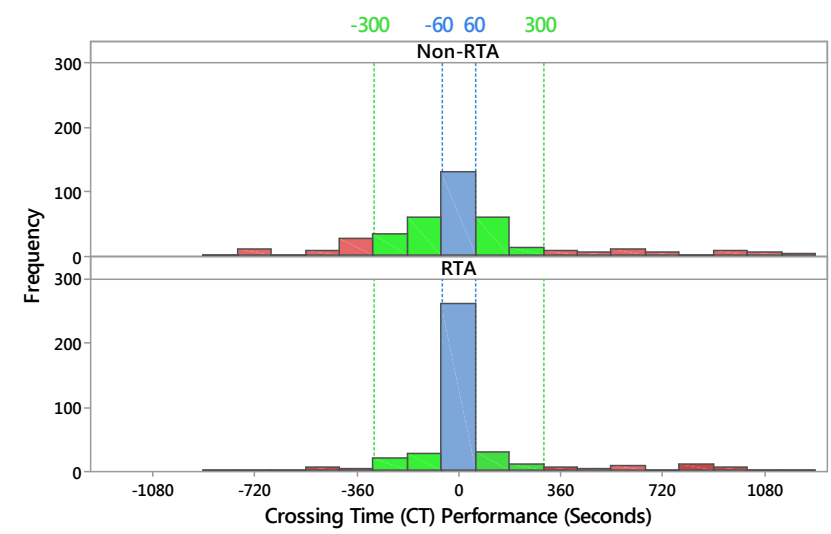

Fig. 8. The histogram of the crossing time performance of the non-RTA assigned aircraft and the RTA assigned aircraft

TABle I. Crossing Time (CT) Performance (Seconds)

\begin{tabular}{cccccc}
\hline \multirow{2}{*}{ Condition } & $\begin{array}{c}\text { Out of } \\
\text { Tolerance } \\
{[-\infty,-300)}\end{array}$ & $\begin{array}{c}\text { Marginal } \\
{[-300,-60)}\end{array}$ & $\begin{array}{c}\text { Targeted } \\
\text { Tolerance Range } \\
{[-60,60]}\end{array}$ & $\begin{array}{c}\text { Marginal } \\
(60,300]\end{array}$ & $\begin{array}{c}\text { Out of } \\
\text { Tolerance } \\
(300,+\infty]\end{array}$ \\
\hline Non-RTA & $12.0 \%$ & $23.7 \%$ & $33.6 \%$ & $18.2 \%$ & $12.4 \%$ \\
RTA & $2.0 \%$ & $12.1 \%$ & $66.9 \%$ & $9.8 \%$ & $9.1 \%$ \\
\hline
\end{tabular}


In order to better understand the impact of departure error, the following analysis seperates the CT performance between airborne aircraft and pre-departure aircraft. The airborne aircraft are the ones that are already in the air at the beginning of the simulation start. Thus, the airborne aircraft did not have to mitigate the departure errors to meet the CT. Instead, they incurred only mild schedule adjustments due to other exempt flights nearby or trajectory uncertainties of the ground based MACS scheduler and actual flight performance trajectories. Hence, the CT performance of the RTA assigned airborne aircraft (79.4\%) was better than the RTA assigned predepartures (53.6\%) with respect to meeting their +/- 60 seconds targeted tolerance range. For meeting the marginal range (+/- 300 seconds), $98.5 \%$ (201 out of 204) of the RTA assigned airborne aircraft and $78.6 \%$ (151 out of 192) of the RTA assigned pre-departures met the marginal range.

To further investigate the effect of RTA on mitigating the departure errors, the scatter plots in Figure 9 was generated. As shown in Figure 9, pre-departures that took-off with EDCT conformance within $+/-300$ seconds in general resulted in CT conformance close to $+/-60$ seconds. For departure errors greater then $+/-300$ seconds, the CT errors were still less than the initial departure errors, suggesting that the RTA was still able to partially mitigate the departure errors.

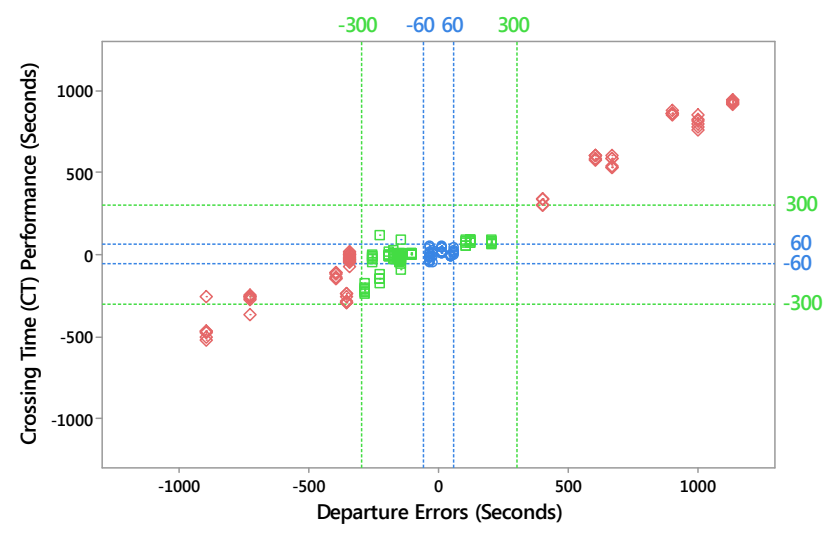

Fig. 9. The scatter plot of crossing time performance (seconds) of the RTA assigned pre-departure aircraft vs. the pre-scripted departure errors (seconds)

Figure 10 shows the CT performance of Non-RTA predeparture aircraft. Comparing the two Figures $9 \& 10$, it can be seen that the CT accuracy improves when the RTA is assigned, particularly for the aircraft (both RTA and NonRTA) departing within the EDCT conformance range (+/- 300 seconds). As expected, CT errors remained as large as the initial departure errors in the Non-RTA condition since the aircraft had no way to correcting the error once the aircraft was airborne.

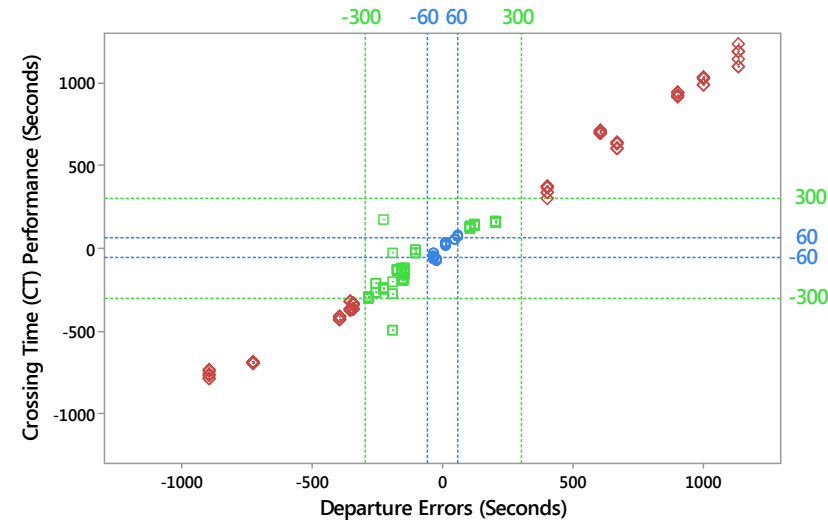

Fig. 10. The scatter plot of crossing time performance (seconds) of the nonRTA assigned pre-departure aircraft vs. the pre-scripted departure errors (seconds)

Table II shows the summary statistics of the pre-scripted departure errors for the RTA assigned pre-departures and Departure Error mitigation (defined as Departure Error - CT performance). The Departure Error mitigation indicates the total amount of departure errors made up by RTA assignment in seconds.

TABLE II. SUMMARY STATISTICS OF TOTAL AMOUNT OF DEPARTURE ERROR MitigATION (SECONDS) AND THE PRE-SCRIPTED DEPARTURE ERRORS (SECONDS)

\begin{tabular}{lccccc}
\hline & Mean & Median & SD & Min. & Max. \\
\hline $\begin{array}{c}\text { Departure } \\
\text { Error } \\
\begin{array}{c}\text { Departure } \\
\text { Error } \\
\text { Mitigation }\end{array}\end{array}$ & 2.4 & -75.0 & 448.1 & -906.0 & 1129.0 \\
\hline
\end{tabular}

B. Results of Hypothesis B: The effect of the heavy wind was minimal on the CT performance in a comparion to the mild wind.

Table III \& IV contain the summary of the CT performance of RTA and Non-RTA assigned aircraft under heavy and mild wind condition respectively. The overall results show no operationally meaningful difference in the CT performance of the RTA assigned aircraft under the influence of different wind severity. Achieving the targeted CT performance (+/-60 seconds tolerance range) of the RTA assigned aircraft under the heavy and the mild wind conditions are $65.1 \%$ (129 out of 198 ) and $68.7 \%$ (136 out of 198) respectively.

TABLE III. CRossing Time (CT) PERFormance (SECONDS) OF THE RTA ASSIGNED AIRCRAFT

\begin{tabular}{cccccc}
\hline $\begin{array}{c}\text { Wind } \\
\text { Severity }\end{array}$ & $\begin{array}{c}\text { Out of } \\
\text { Tolerance } \\
{[-\infty,-300)}\end{array}$ & $\begin{array}{c}\text { Marginal } \\
{[-300,-60)}\end{array}$ & $\begin{array}{c}\text { Targeted } \\
\text { Tolerance Range } \\
{[-60,60]}\end{array}$ & $\begin{array}{c}\text { Marginal } \\
(60,300]\end{array}$ & $\begin{array}{c}\text { Out of } \\
\text { Tolerance } \\
(300,+\infty]\end{array}$ \\
\hline Heavy & $1.5 \%$ & $14.1 \%$ & $65.1 \%$ & $8.6 \%$ & $10.6 \%$ \\
Mild & $2.5 \%$ & $10.1 \%$ & $68.7 \%$ & $11.1 \%$ & $7.6 \%$ \\
\hline
\end{tabular}


TABlE IV. CRossing Time (CT) PERformance (SECONDS) OF THE NON-RTA ASSIGNED AIRCRAFT

\begin{tabular}{cccccc}
\hline $\begin{array}{c}\text { Wind } \\
\text { Severity }\end{array}$ & $\begin{array}{c}\text { Out of } \\
\text { Tolerance } \\
{[-\infty,-300)}\end{array}$ & $\begin{array}{c}\text { Marginal } \\
{[-300,-60)}\end{array}$ & $\begin{array}{c}\text { Targeted } \\
\text { Tolerance Range } \\
{[-60,60]}\end{array}$ & $\begin{array}{c}\text { Marginal } \\
(60,300]\end{array}$ & $\begin{array}{c}\text { Out of } \\
\text { Tolerance } \\
(300,+\infty]\end{array}$ \\
\hline Heavy & $12.6 \%$ & $18.7 \%$ & $33.8 \%$ & $19.7 \%$ & $15.2 \%$ \\
Mild & $11.6 \%$ & $28.8 \%$ & $33.3 \%$ & $16.7 \%$ & $9.6 \%$ \\
\hline
\end{tabular}

Figure 11 shows the CT performance per traffic flows (North, South, and West Flows). The aircraft are categorized as "airborne" or "pre-departure" depending on whether they were already in flight at the beginning of the simulation. In general, the effect of wind severity on the CT performance was minimal. However, differences between heavy and mild winds were observed for specific flows, such as the West Flow during both RTA and Non-RTA conditions for airborne aircraft.

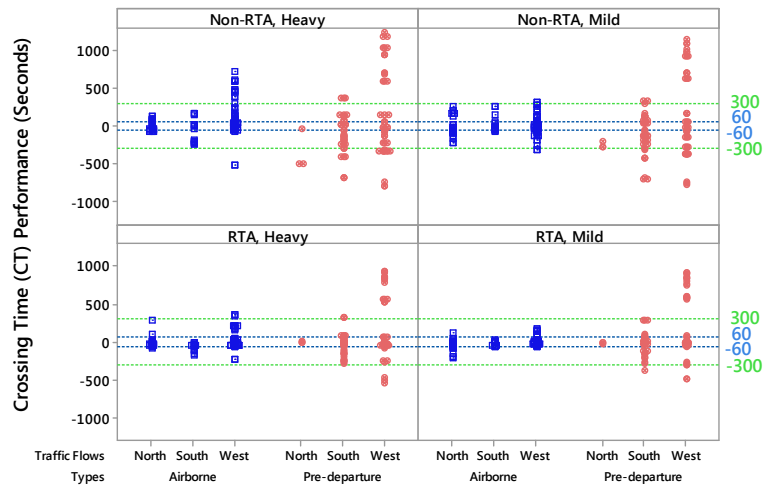

Fig. 11. The scatter plot of crossing time performance (seconds) of both the RTA and non-RTA assigned aircraft per flow

C. Hypothesis C: Flight distance of RTA assigned aircraft affects Crossing Time performance.

The CT performance of Non-RTA and RTA assigned aircraft as a function of flight distance (Miles) was examined. Figure 12 indicates the CT performance of the RTA and NonRTA assigned aircraft in a relation to the Flight Distance (Miles) per traffic flows (North, South, and West flows), where the flight distance is the distance that the aircraft flew from the point when the RTA was assigned to the point that the aircarft crossed the FEA. In the figure, each CT performance is color-coded by the aircraft category (airborne or pre-departure).

In the figure, it is observed that both the RTA and NonRTA assigned aircraft's CT performance for the North flow is very accurate, as the CT performance stayed within the maginal region and delivered close to the target conformance (+/- 60 seconds). By comparing the results of the Non-RTA to the RTA condition, it can be inferred that no significant amount of mitigation was needed by assigning RTA. It is observed that the South flow had a relatively shorter flight distance for both pre-departure and airborne. The CT performance of both RTA and Non-RTA assigned aircraft for the South flow stayed within the target and the marginal tolerance range. The benefit of assigning RTA can be seen most clearly for the West flow as the RTA assigned airborne aircraft with the long flight distance show better CT performance than the Non-RTA assigned aircraft.

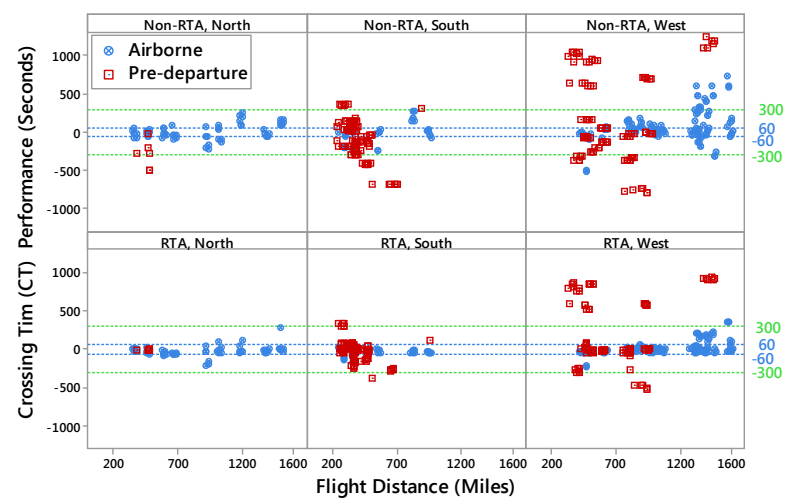

Fig. 12. The scatter plot of crossing time performance (seconds) of both the RTA and non-RTA assigned pre-departure aircraft vs. flight distance

To further investigate the effecft of the flight distance on the CT performance of the RTA assigned aircraft, Figure 13 was constructed.

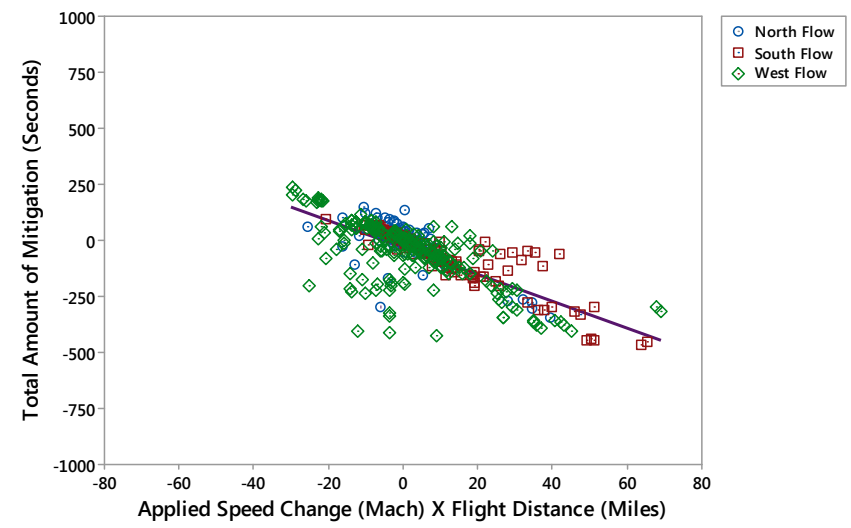

Fig. 13. The Scatter plot of total amount of mitigation (seconds) of the RTA assigned aircraft based on the speed adjustment applied and the flight distance (miles).

Figure 13 above shows a trend describing the total amount of mitigation (Seconds) made through the applied speed change (Mach) in average multiplied by the flight distance (Miles) due to assigning the RTA, where the total amount of mitigation indicates how much of the difference between initial ETA at the moment when the RTA was assigned and the target CT made up by applying the RTA. The applied speed change is the average speed adjustment made by applying the RTA to the initial cruise speed that the aircraft was flying. In the figure, it can be observed that the amount of mitigation increases as either/or both applied speed adjustment and flight distance increase. 
D. Hypothesis D: Crossing Time performance of the RTA assigned aircraft decreased as a function of increase in wind forecast errors.

Tables V \&VI summarize the CT performance of the RTA assign Aircraft under heavy and mild wind conditions. The data show a decrease in CT performance as wind forecast errors increases; however, the effect seems to be very modest.

TABLE V. THE EFFECT OF WIND FORECAST ERRORS ON CROSSING TIME (CT) PERFormANCE (SECONDS) OF RTA AsSignED AirCRAFT (HEAVY WIND)

\begin{tabular}{|c|c|c|c|c|c|}
\hline $\begin{array}{c}\text { Wind } \\
\text { Forecast } \\
\text { Errors }\end{array}$ & $\begin{array}{c}\text { Out of } \\
\text { Tolerance } \\
{[-\infty,-300)}\end{array}$ & $\begin{array}{l}\text { Marginal } \\
{[-300,-60)}\end{array}$ & $\begin{array}{c}\text { Targeted } \\
\text { Tolerance } \\
\text { Range } \\
{[-60,60]}\end{array}$ & $\begin{array}{l}\text { Marginal } \\
(60,300]\end{array}$ & $\begin{array}{c}\text { Out of } \\
\text { Tolerance } \\
(300,+\infty]\end{array}$ \\
\hline 1 & $1.5 \%$ & $10.6 \%$ & $71.2 \%$ & $7.6 \%$ & $10.6 \%$ \\
\hline 2 & $1.5 \%$ & $13.6 \%$ & $66.7 \%$ & $9.1 \%$ & $10.6 \%$ \\
\hline 5 & $1.5 \%$ & $18.2 \%$ & $62.1 \%$ & $9.1 \%$ & $10.6 \%$ \\
\hline
\end{tabular}

TABLE VI. THE EFFECT OF WIND FORECAST ERRORS ON CROSSING TIME (CT) PERFormANCE (SECONDS) OF RTA AsSignED AIRCRAFT (MILD Wind)

\begin{tabular}{|c|c|c|c|c|c|}
\hline $\begin{array}{c}\text { Wind } \\
\text { Forecast } \\
\text { Errors }\end{array}$ & $\begin{array}{c}\text { Out of } \\
\text { Tolerance } \\
{[-\infty,-300)}\end{array}$ & $\begin{array}{l}\text { Marginal } \\
{[-300,-60)}\end{array}$ & $\begin{array}{c}\text { Targeted } \\
\text { Tolerance } \\
\text { Range } \\
{[-60,60]}\end{array}$ & $\begin{array}{l}\text { Marginal } \\
(60,300]\end{array}$ & $\begin{array}{c}\text { Out of } \\
\text { Tolerance } \\
(300,+\infty]\end{array}$ \\
\hline 1 & $3.0 \%$ & $7.6 \%$ & $74.2 \%$ & $7.6 \%$ & $7.6 \%$ \\
\hline 2 & $1.5 \%$ & $13.6 \%$ & $66.7 \%$ & $12.1 \%$ & $7.6 \%$ \\
\hline 5 & $3.0 \%$ & $9.1 \%$ & $69.7 \%$ & $12.1 \%$ & $7.6 \%$ \\
\hline
\end{tabular}

\section{DISCUSSION}

The study was conducted to explore the use of RTA as a control mechanism to mitigate the uncertainty that may impede effective delivery of the planned traffic demand into TBFM under IDM concept. Although the results of the study show descriptive differences in the CT performance of the RTA assigned aircraft due to the effect of the two selected factors (i.e. the wind severity and the wind forecast errors), it was found that more accurate traffic delivery (+/- 60 seconds target tolerance range) can be achieved by assigning RTA, regardless of the influence of the selected factors. The results show that the wind severity has less impact on the overall performance of the CT conformance of the traffic in a comparison between RTA and Non-RTA condition. This was in large part due to the variability of wind across each flow (tail wind or head wind). The wind characteristics affect each aircraft differently based on its location. In addition, the results suggest that the greater wind forecast errors within FMS can reduce CT performance, especially in heavy winds, but again the impact of the errors were small. Although assigning RTA delivered more accurate CT performance, Non-RTA condition might also deliver a "reasonable" CT performance if achieving the $+/-300$ seconds marginal tolerance range was sufficient for pre-conditioning the traffic into TBFM.

Several limitations for using the RTA were observed. Each RTA assigned aircraft was restricted to fly within the speed limit that was specified and also each aircraft has its maximum speed limitation. Hence, depending on the initial speed when the RTA was assigned, the room for the speed adjustment was limited and may forbid the aircraft to speed up or slow down further. In addition, the flight distance (i.e. the flight distance after RTA is assigned until aircraft crosses the FEA) for the pre-departure aircraft were relatively shorter than the airborne aircraft because the aircraft have to reach the top-of-climb to receive the RTA advisory. Hence, although the improvement of the CT accuracy could be achieved by assigning RTA, meeting the target tolerance range (+/- 60 seconds) or marginal range (+/- 300 seconds) was mainly driven by whether the pre-departure departed within the EDCT conformance range ( $+/-5$ minutes).

\section{CONCLUSION AND GOING FORWARD}

After conducting the study, the following conclusions are made:

A. The traffic delivery accuracy improvement was achieved by assigning RTA to the aircraft.

B. The difference in the wind severity of the selected heavy and the mild wind condition did not show significant impact on the CT performance.

C. Flight distance of RTA assigned aircraft affected the CT performance.

D. The CT performance of the RTA assigned aircraft degraded modestly as a function of increase in wind errors (Wind forecast errors).

Based on the lessons-learned from this study, future studies can follow. The future studies can verify the effect of accurate CT performance by using RTA on actual traffic throughput into EWR under the IDM concept. The results of this study indicate that the RTA brings more accuracy in traffic delivery against Non-RTA, particularly if the target CT is +/- 60 seconds. The results of such future studies can help identify the threshold value for the target or marginal CT tolerance range allowing the effective operation of IDM, which can help determine whether such accurate delivery achieved by RTA assignment is necessary for the concept. Once the need of using RTA for the IDM concept is fully verified, the subsequent studies can follow to explore additional benefit of RTA, such as how RTA helps maintain the schedules that are produced over long ranges and adds values in achieving more stable and predictable scheduling operations. Also, various operational approaches of using RTA under IDM operation, such as how IDM operations differ when RTA is no longer an advisory but an actual air traffic clearance, can be conducted. In addition, the results of this study show the limitation of RTA in how much mitigation it can make under the selected condition. Hence, more exploration on new control mechanisms to mitigate the uncertainty greater than what could be achieved by assigning RTA should follow as well.

\section{ACKNOWLEDGMENT}

The authors would like to thank the lab personnel at Airspace Operations Lab (AOL) at NASA Ames Research 
Center and all of our airspace Subject Matter Experts for the invaluable contributions to the IDM RTA concept.

\section{REFERENCES}

[1] N.M. Smith, and et al. "Integrated Demand Management: Coordinating Strategic and Tactical Flow Scheduling Operations." 16th AIAA Aviation Technology, Integration, and Operations Conference. 2016.

[2] E.D. Antony, and P.U. Lee. "Analyzing Double Delays at Newark Liberty International Airport." In AIAA Aviation Technology, Integration, and Operations Conference. June 2016.

[3] S.J. Landry, and A. Villanueva. "Mitigating the effect of demand uncertainty due to departures in a national timebased metering system." 7th AIAA Aviation Technology, Integration, and Operations Conference. 2007.

[4] A. Donaldson, and R. Hansman. "Capacity improvement potential for the new york metroplex system." In 10th AIAA Aviation Technology, Integration and Operations (ATIO) Conference and AIAA/ISSMO Multidisciplinary Analysis Optimization (MAO), Fort Worth, TX. 2010.

[5] H.S. Yoo, P. Lee, and E. Palmer. "Improving departure throughput by dynamically adjusting inter-arrival spacing." In 2014 IEEE/AIAA 33rd Digital Avionics Systems Conference (DASC), pp. 1A4-1. IEEE, 2014.
[6] S. Kamine, S. Tien, and W. Cooper. "Analysis of AFP Route-Outs in Preparation for CTOP Post-Implementation Assessment." In AIAA Aviation Technology, Integration, and Operations Conference, American Institute of Aeronautics and Astronautics. 2013.

[7] S.J. Landry, T. Farley, T. Hoang, and B. Stein, A distributed scheduler for air traffic flow management. Journal of Scheduling, 2012, pp.537551.

[8] T. Prevot, "Exploring the many perspectives of distributed air traffic management: The Multi Aircraft Control System MACS." In Proceedings of the HCI-Aero, 2002, pp. 149-154.

[9] S. Park, S. Vaddi, and J. Kwan. "Next Generation Flight Management System Simulator." In AIAA Modeling and Simulation Technologies Conference. 2016, pp. 425.

[10] T.G. Reynolds, Y. Glina, S.W. Troxel, and M. McPartland. "Establishing Wind Information Needs for Four Dimensional Trajectory-Based Operations." In 1st International Conference on Interdisciplinary Science for Innovative Air Traffic Management, Daytona Beach, FL. 2012.

[11] T.G. Reynolds, Y. Glina, S.W. Troxel, and M.D. McPartland. Wind Information Requirements for NextGen Applications Phase 1: 4DTrajectory Based Operations (4D-TBO). No. ATC-399. FAA-ATC-399, Massachusetts Institute of Technology Lincoln Laboratory, 2013. 